\title{
Prephase rituximab/prednisone therapy and aging-related, proinflammatory cytokine milieu in older, vulnerable patients with newly diagnosed diffuse large B-cell lymphoma
}

Haematologica 2022

Volume 107(5):1144-1152

\section{Correspondence:}

PAUL A. HAMLIN

hamlinp@mskcc.org

Received: March 6, 2021.

Accepted: July 12, 2021.

Pre-published: July 22, 2021.

https://doi.org/10.3324/haematol.2021.278719

(C)2022 Ferrata Storti Foundation

Material published in Haematologica is covered by copyright. All rights are reserved to the Ferrata Storti Foundation. Use of published material is allowed under the following terms and conditions:

https://creativecommons.org/licenses/by-nc/4.0/legalcode. Copies of published material are allowed for personal or internal use. Sharing published material for non-commercial purposes is subject to the following conditions:

https://creativecommons.org/licenses/by-nc/4.0/legalcode, sect. 3. Reproducing and sharing published material for commercial purposes is not allowed without permission in writing from the publisher.

\section{Richard J. Lin, ${ }^{1,2 *}$ Colette N. Owens, ${ }^{1,2 *}$ Esther Drill, ${ }^{3}$ Augustine lannotta, ${ }^{1}$ Mayan Oliveros, ${ }^{1}$ Dylan L. Schick, ${ }^{1}$ Ariela Noy, ${ }^{1,2}$ John F. Gerecitano, ${ }^{1,2}$ Pamela R. Drullinsky, ${ }^{1,2}$ Philip C. Caron, ${ }^{1,2}$ Anita Kumar, ${ }^{1,2}$ Matthew J. Matasar, ${ }^{1,2}$ Craig Moskowitz, ${ }^{1,2}$ Beatriz Korc-Grodzicki, ${ }^{2,4}$ Andrew D. Zelenetz, ${ }^{1,2}$ Gilles A. Salles, ${ }^{1,2}$ and Paul A. Hamlin ${ }^{1,2}$}

${ }^{1}$ Department of Medicine, Division of Hematologic Malignancies, Memorial Sloan Kettering Cancer Center; ${ }^{2}$ Department of Medicine, Weill Cornell Medical College; ${ }^{3}$ Epidemiology and Biostatistics, Memorial Sloan Kettering Cancer Center and ${ }^{4}$ Department of Medicine, Geriatrics Service, Memorial Sloan Kettering Cancer Center, New York, NY, USA

${ }^{\star} R J L$ and $C N O$ contributed equally as co-first authors.

\section{ABSTRACT}

$\mathrm{D}$ iffuse large B-cell lymphoma (DLBCL) predominantly affects older adults with suboptimal therapeutic outcomes due to increased treatment-related mortality and toxicities in vulnerable patients, clinically defined by geriatric impairments such as functional limitation, multimorbidity, or cognitive deficits. In this prospective pilot study, we evaluated a rituximab/prednisone prephase treatment strategy in 33 older, vulnerable patients with newly diagnosed DLBCL, defined by either age $\geq 70$ years or age $60-70$ years with Karnofsky performance scale (KPS) $<80$. A single dose of rituximab $375 \mathrm{mg} / \mathrm{m}^{2}$ between 3-10 days and oral prednisone for at least 5 days prior to the first dose of chemoimmunotherapy was administered. All patients completed prephase treatment and all but one commenced anthracycline-based chemoimmunotherapy. Only one early cycle death occurred. Toxicity events, defined by either unplanned hospitalization, unplanned dose reduction/delay, or chemotherapy discontinuation, occurred in 22 patients (67\%). Sixteen patients (48\%) experienced grade 3 or higher non-hematologic toxicities and/or grade 4 or higher hematologic toxicities. With a median follow-up of 4.4 years, both 5 -year progression-free survival and overall survival were at $81 \%(95 \%$ confidence interval: 69-96). Importantly, we found that phenotypic impairments in basic and instrumental activities of daily living, physical function, mobility, KPS, and Cancer and Aging Research Group chemotherapy toxicity risk score were significantly associated with senescence-associated, proinflammatory cytokine milieu which was readily reversed with prephase treatment, potentially explaining its clinical effectiveness. Prephase therapy with rituximab/prednisone should be considered for all older, vulnerable DLBCL patients prior to curative intent, anthracycline-based chemoimmunotherapy. This trial was registered as clinicaltrials gov. Identifier: NCT 89028394.

\section{Introduction}

Diffuse large B-cell lymphoma (DLBCL) disproportionally affects older patients and improving their therapeutic outcomes remains an unmet medical need. Epidemiologic studies have shown that even in the rituximab era, many older patients either do not receive or receive suboptimal dose and/or duration of chemoimmunotherapy to achieve a curative intent. ${ }^{1,2}$ While the biology of disease may be more aggressive, ${ }^{3}$ older patients commonly have multimorbidity, functional and/or cognitive impairment, or overt frailty that limits the delivery of upfront, cur- 
ative chemoimmunotherapy. ${ }^{4,5}$ Moreover, if not adequately addressed, these non-oncologic geriatric issues may exacerbate treatment-related toxicities, trigger functional decline, and adversely impact subsequent therapies such as hematopoietic cell transplantation and cellular therapy. ${ }^{4-6}$ Therefore, it is essential that older patients with newly diagnosed DLBCL receive adequate assessment and management of their aging-related issues.

Biologically, inferior outcomes in older non-Hodgkin lymphoma (NHL) patients may result from preexisting geriatric deficits, the lymphoma itself, or treatment-related toxicities. Induction chemoimmunotherapy may worsen health status when it results in toxicities or improve it by controlling disease related impairments. The German Non-Hodgkin Lymphoma Study Group (DSHNHL) found that after the initiation of the NHL-B2 trial, toxic mortality was most common in the first and second cycle of therapy. ${ }^{7}$ They subsequently pioneered a strategy of "prephase" therapy with prednisone $100 \mathrm{mg}$ daily for 5-7 days with or without vincristine $1 \mathrm{mg}$ single dose prior to the initiation of full dose combination chemotherapy. This was incorporated into the latter part of the NHL-B2 trial, RICOVER60 trial, and the LYSA group LNH097B trial with fewer toxic deaths reported. ${ }^{7-9}$ The mechanism of this effect has not been examined in detail, although it is thought that prephase therapy improves functional status and physiologic reserve by reducing tumor burden. However, although vincristine is delivered by a simple 10-minute intravenous push, it is among the more toxic agents with significant risks of neuropathy and constipation resulting in its frequent dose reduction or omission. ${ }^{10}$

Geriatric assessment (GA) is increasingly incorporated into the care of older cancer patients to help guide treatment decision-making, predict toxicities, and manage non-oncologic geriatric issues. Multidimensional GA identifies otherwise unrecognized health problems among unselected older patients beyond traditional Karnofsky performance scale (KPS) and includes function status, comorbidity, mobility, cognition, nutrition, and psychosocial status. ${ }^{11,12}$ A largely self-administered GA instrument, the Cancer Aging Research Group (CARG) chemotherapy toxicity risk score incorporates 11 variables to predict high-grade, chemotherapy-related toxicities for older solid tumor patients. ${ }^{13,14}$ Several GA domains have been examined in small cohort studies of lymphoma patients, yet it remains unclear how they could be integrated into and improve outcomes in the context of curative intent chemoimmunotherapy. ${ }^{15}$

It has been long postulated that the mechanism underlying phenotypic and functional aging is related to perturbations in several biochemical and cellular pathways. ${ }^{16}$ One of them is cellular senescence, a state of stable growth arrest once cells are subjected to significant stress and have accumulated enough DNA damage. ${ }^{17}$ Senescence cells create a highly dynamic and persistent program of senescence-associated secretory phenotype (SASP), consisting of abundant secretion of proinflammatory proteins into the tissue microenvironment that modulates cancer immune surveillance and therapeutic response. ${ }^{18}$ Identification of important SASP components may generate novel targets to restore immune therapy responsiveness and enhance treatment outcomes. ${ }^{19}$ In this prospective pilot study, we examine the feasibility and safety of a novel prephase treatment with rituximab/prednisone for older, vulnerable patients with newly diag- nosed DLBCL and its impact on meaningful toxicity outcomes, CARG-based GA measures, and the SASP-related, proinflammatory cytokine milieu.

\section{Methods}

\section{Trial design and schema}

We conducted a pilot study of rituximab/prednisone prephase treatment for older patients with newly diagnosed DLBCL prior to curative intent, anthracycline-based chemoimmunotherapy within our larger prospective observational study (Figure 1). Patients were eligible if they were aged 70 years or older, or were 60-70 years old with KPS $<80$. The protocol was approved by the Institutional Review Board at the Memorial Sloan Kettering Cancer Center and conducted according to the Declaration of Helsinki. All patients received a single dose of rituximab $375 \mathrm{mg} / \mathrm{m}^{2}$ given between 3 and 10 days and oral prednisone for at least 5 days during the 14 days prior to the first cycle. The preferred prednisone dose was $100 \mathrm{mg}$ daily for 7 days with minimal allowable dose of $50 \mathrm{mg}$ daily for 5 days.

\section{Geriatric assessment}

CARG-based GA was performed at baseline, following prephase treatment and before the first cycle, and prior to each subsequent cycle as previously described. ${ }^{13,14}$ The CARG chemotherapy risk calculator provided both a risk score (range, 0-19) and a corresponding absolute chemotherapy-toxicity risk percentage. The clinician portion of the GA included baseline patient and disease characteristics, clinician-rated KPS, memory screening test short Blessed Orientation-Memory-Concentration (BOMC), ${ }^{20}$ Timed Up and Go (TUG), and Mini-Nutrition Assessment (MNA). ${ }^{21}$

\section{Outcome and toxicities assessment}

The primary outcome was the composite of toxicity or severe toxicity events (TE). TE was defined as any of the following: i) hospitalization during or within 30 days following chemotherapy; ii) dose delay or reduction to a dose intensity $\leq 80 \%$ of the planned dose intensity; iii) discontinuation of chemotherapy due to toxicity. Severe TE (STE) was defined as the occurrence of either i) or iii) above. Secondary toxicity endpoints were: i) grade 3 or higher non-hematologic toxicity; ii) grade 4 or higher hematologic toxicity. Toxicity was graded according to CTCAE v4.0.3.

\section{Proinflammatory cytokine analysis}

Immunoassays of the levels of proinflammatory cytokines were batch performed in duplicate on singly thawed plasma samples on a commercial multiplex cytokine panel including IL6, IL-1b, IL-2, IL-4, IL-5, IL-8, IL-10, IL-12, TNF- $\alpha$, and IFN- $\gamma$ (Miso Discovery Laboratory). These cytokines were chosen given commercial availabilities on multiplex plates and technical expertise at our institution.

\section{Statistical analyses}

In order to compare pre- and posttreatment GA scales and cytokine (CK) levels, one-sided pairwise Wilcoxon tests were used. CK levels were log-transformed before analyses. Associations between baseline GA, $\Delta \mathrm{GA}$, baseline $\mathrm{CK}, \Delta \mathrm{CK}$ and toxicities were assessed with one-sided Wilcoxon rank sum tests. Associations between baseline GA, $\Delta \mathrm{GA}$, baseline CK, $\Delta \mathrm{CK}$ and stage were assessed with two-sided Wilcoxon rank sum tests. Associations between toxicities and stage were assessed with Fishers' Exact test. Correlations between baseline 
GA measures and CK levels were assessed with the Spearman's rank correlation coefficient. Progression-free survival (PFS) was calculated from treatment initiation to disease progression or death. Overall survival (OS) was calculated from diagnosis to death. All tests were corrected for multiple testing with the false discovery rate (q-value) at a significance level of 0.05 . Statistical analyses were conducted in $\mathrm{R}$ Version 4.0.0.

\section{Results}

\section{Patient characteristics}

Baseline characteristics are summarized in Table 1. The cohort consisted of 33 patients with a median age of 75 years (range, 65-87) and ten patients $(30 \%)$ were 80 years old or older. Nearly two thirds of patients were stage III and IV at diagnosis and more than half had intermediate2/high risk-disease based on age-adjusted International Prognostic Index (AA-IPI). All patients completed prephase treatment with rituximab and prednisone, with 26 patients full $100 \mathrm{mg}$ dose and 7 patients $50 \mathrm{mg}$ doses. One patient did not receive chemoimmunotherapy following prephase treatment due to rapid disease progression. For the 32 patients who initiated chemoimmunotherapy, 23 of 25 patients $(92 \%)$ completed all six planned cycles; six of six completed all four planned cycles; and there was one early death due to rapid disease progression.

Baseline GA revealed that 23 patients $(70 \%)$ had intermediate to high comorbidity burden. There were significant functional limitations in $\mathrm{ADL}$ (median 65; range, 0100), IADL (median 14, range, 0-14), and social activities (median 50; range 25-75). The median TUG time was 11.31 seconds (range, $5-40$ ), with $<10$ seconds denoting no mobility limitation. The median MNA score was 23 (range, 10.5-30), with 15 patients (45\%) considered at risk for malnutrition or malnourished (score $>24$ ). The median CARG chemotherapy toxicity risk score was 10 (range, 6$17)$, corresponding to an absolute, grade $3+$ chemotherapy-related toxicity risk of $54 \%$ (range, 32-89), both in the high-risk category.

\section{Outcomes and toxicities}

As shown in Figure 2, with a median follow-up of 4.4 (range, 0.4-5.7) years, both 5-year PFS and OS for the cohort were $81 \%$ (95\% confidence interval [CI]: 69-96). PFS and OS according to AA-IPI is also shown in Figure 2. Among stage III/IV patients $(n=20)$, the 5 -year PFS and OS were both $74 \%$ ( $95 \%$ CI: 57-97, data not shown). Six patients died, including four from relapse/progression of disease and two while in remission. We summarized toxic events and high-grade CTATE toxicities through all treatment cycles in Table 2. TE and STE occurred in 22 patients $(67 \%)$ and 12 patients $(36 \%)$, respectively. The majority of TE was dose reduction/delay, occurring in 19 patients $(58 \%)$. There were seven hospitalizations following cycle 1 . Grade 3+ non-hematologic toxicities occurred in 16 patients $(48 \%)$. The total number of grade $3+$ nonhematologic toxicity was 26 , including ten infections (3 following cycle 1), five cardiac toxicities, three electrolyte/metabolic toxicities, three gastrointestinal/nutritional toxicities, two hemorrhage/thrombosis, one infusion reaction, and one psychiatric illness. Grades 3+ and $4+$ hematologic toxicities occurred in 23 patients $(70 \%)$ and six patients $(18 \%)$, respectively. Most toxicities, 17 $(65 \%)$, was in cycle $1-3$. In total, 16 patients $(48 \%)$ had at least one grade $3+$ non-hematological toxicity or grade $4+$ hematologic toxicity. Toxicity events did not differ significantly by stage of disease at diagnosis (Online Supplementary Table S1).

\section{Geriatric assessment and senescence-associated secretory phenotype-associated proinflammatory cytokines}

We examined the senescence-associated, proinflammatory cytokine milieu in our cohort of older patients and its relationship to clinical geriatric impairments. As shown in Figure 3 and the Online Supplementary Table S2, we found that levels of several proinflammatory cytokines were associated with individual geriatric impairment in older lymphoma patients. Specifically, elevated interleukin (IL)2 , IL-6, IL-10, and TNF- $\alpha$ levels were significantly associated with reduced KPS, functional limitation measured by

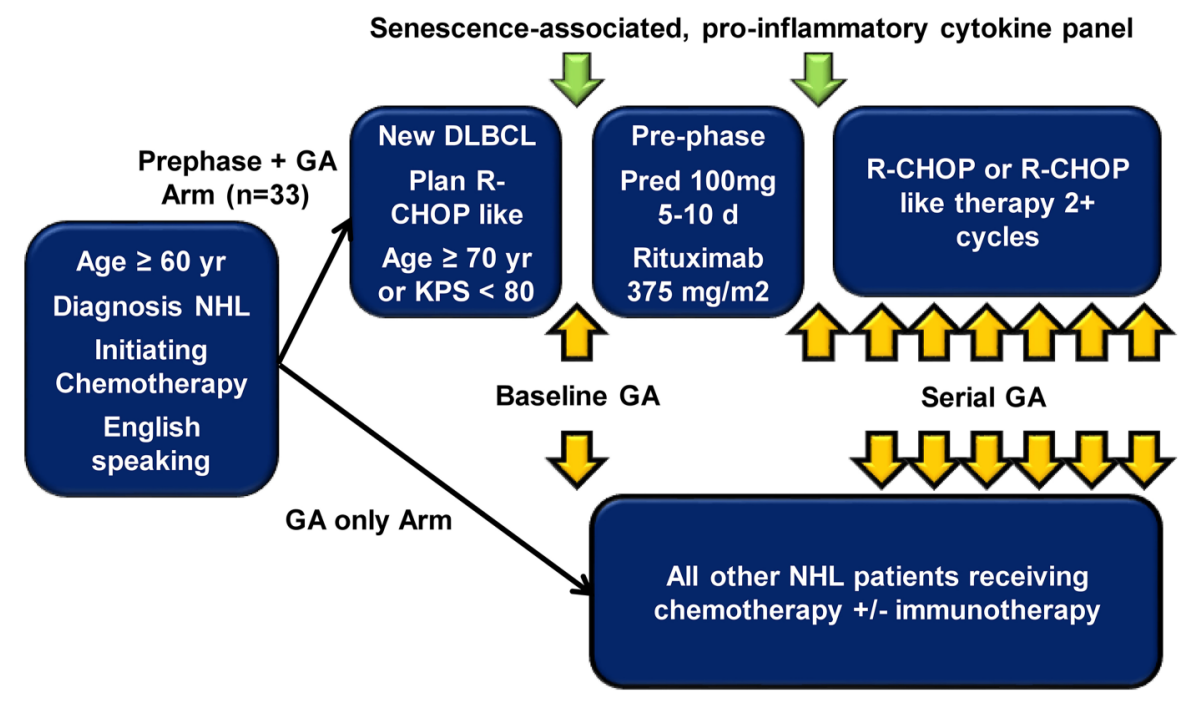

Figure 1. Study design. Prephase pilot embedded within a prospective, large cohort study of geriatric assessment in older patients with newly diagnosed non-Hodgkin lymphoma. DLBCL: diffuse large B-cell lymphoma; GA: geriatric assessment; NHL: non-Hodgkin lymphoma; KPS: Karnofsky performance scale; R-CHOP: rituximab, cyclophosphamide, doxorubicin hydrochloride, vincristine sulfate, and prednisone. 
$\mathrm{ADL}$ and IADL, and social activity limitation. They were also significantly associated with increased TUG time, CARG chemotherapy toxicity risk, and baseline LDH level.

We next examined the impact of prephase treatment on GA measures and CK levels. As shown in Table 3, prephase therapy led to significant reduction in levels of IL-10 ( $q=0.033)$, IL-4 ( $q=0.04)$, IL-6 ( $q=0.01)$, and TNF- $\alpha$ $(q=0.01)$. The magnitude of changes was most pronounced for TNF- $\alpha$ and IL-6 (Online Supplementary Figure S1). Prephase therapy did not lead to significant changes in GA measures within the 2 -week period (Table 3 ),

Table 1. Baseline characteristics.

\begin{tabular}{|c|c|}
\hline & Total cohort $(\mathrm{N}=33)$ \\
\hline Age, years, median (range) & $75(65-87)$ \\
\hline Female sex, n (\%) & $20(61)$ \\
\hline \multicolumn{2}{|l|}{ Stage, n (\%) } \\
\hline $\mathrm{I} / \mathrm{II}$ & $13(39)$ \\
\hline III/IV & $20(61)$ \\
\hline \multicolumn{2}{|l|}{ Cell of origin (n=31), Hans, $n(\%)$} \\
\hline $\mathrm{GCB}$ & $18(58)$ \\
\hline Non-GCB & $13(42)$ \\
\hline \multicolumn{2}{|l|}{ Histology, n (\%) } \\
\hline De novo & $29(88)$ \\
\hline Transformed follicular & $3(9)$ \\
\hline Richter's transformation & $1(3)$ \\
\hline \multicolumn{2}{|l|}{ Age-adjusted IPI, n (\%) } \\
\hline Low/Int-1 & $14(42)$ \\
\hline Int-2/High & $19(58)$ \\
\hline \multicolumn{2}{|l|}{ Induction regimen, $\mathrm{n}(\%)$} \\
\hline R-CHOP & $29(91)$ \\
\hline R-mini-CHOP & $1(3)$ \\
\hline R-EPOCH & $2(6)$ \\
\hline No treatment & 1 \\
\hline \multicolumn{2}{|l|}{ Comorbidity, n (\%) } \\
\hline Low & $10(30)$ \\
\hline Intermediate & $17(52)$ \\
\hline High & $6(18)$ \\
\hline Clinician rated KPS, median (range) & $80(40-100)$ \\
\hline Patient rated KPS, median (range) & $80(40-100)$ \\
\hline ADL score, median (range) & $65(0-100)$ \\
\hline IADL score, median (range) & $14(2-14)$ \\
\hline Activity limitation score, median (range) & $50(25-75)$ \\
\hline Number of falls last 6 months, median (range) & $0(0-9)$ \\
\hline TUG in seconds, median (range) & $11.31(5-40)$ \\
\hline Cognition score (BOMC), median (range) & $2(0-10)$ \\
\hline Mini-nutritional assessment, median (range) & $23(10.5-30)$ \\
\hline CARG score, median (range) & $10(6-17)$ \\
\hline CARG \% risk, median (range) & $54(32-89)$ \\
\hline
\end{tabular}

GCB: germinal center B-cell type; IPI: international prognostic index; R-CHOP: Rituximab, Cyclophosphamide, Doxorubicin hydrochloride, Vincristine sulfate, Prednisone; R-EPOCH: Rituximab, Etoposide phosphate, Prednisone, Vincristine sulfate, Cyclophosphamide, Doxorubicin hydrochloride; KPS: Karnofsky performance scale; ADL: activities of daily living; IADL: instrumental activities of daily living; TUG: timed-get-up and go; BOMC: Blessed Orientation Memory Concentration; CARG: cancer and aging research group. although there was a trend of improvement in clinician rated KPS and CARG chemotherapy toxicity risk score prior to correction for multiple testing. Neither baseline or changes in GA measures or CK levels differed by early versus later stage of disease (Online Supplementary Tables S3 and 54 ).

\section{Association of geriatric assessment and cytokines with outcomes}

Finally, we examined the association of both baseline and changes in GA measures and CK levels following prephase therapy with outcomes. As shown in Table 4, we did not find a significant association of any baseline or changes in GA measures with the development of TE, although there was a trend toward significance for baseline CARG risk score $(P=0.040)$ and the absolute toxicity risk percentage $(P=0.019)$ prior to correction for multiple testing. Similarly, there was no significant association between baseline or changes in cytokine levels with the development of TE, although there was a trend toward significance for baseline IL-10 $(P=0.012)$ and IL-13 $(P=0.006)$ prior to correction for multiple testing (Online Supplementary Table S5). Due to the small number of PFS/OS events $(n=6)$, we did not assess the association of GA measures and CK levels with these outcomes.

\section{Discussion}

Improving outcomes for older, vulnerable patients with aggressive lymphoma has remained a challenge over the last few decades. In this study, we examined a novel rituximab/prednisone prephase therapy for older, vulnerable DLBCL patients prior to curative, anthracycline-based chemoimmunotherapy. This prephase therapy was feasible with acceptable toxicity profiles. Most patients in this vulnerable cohort completed the planned 4-6 cycles of treatment, although dose reduction/delays were common. Although conclusions are limited by the pilot nature of the study with small sample size, the favorable 5-year survival of over $80 \%$ was comparable to selected historical

Table 2. Toxicity events and high-grade toxicities.

Categony of events
Number of
patients $(\%)$

Toxicity events (unplanned hospitalization,

$22(67)$

chemotherapy discontinuation, and/or dose

reduction/delay)

Severe toxicity events

$12(36)$

(unplanned hospitalization and/or

chemotherapy discontinuation)

Early induction death (prior to cycle 3 )

Category of toxicities

Grade 3+ hematologic toxicitie

Grade 4+ hematologic toxicities

Grade 3+ non-hematologic toxicities

Grade $3+$ non-hematologic toxicities plus

$16(48)$

Grade 4+ hematologic toxicities

16 (48)

Total numbers of grade 3+ non-hematologic toxicities: infection (10); cardiac (5); electrolyte/metabolic (3); gastrointestinal/nutrition (3); bleeding/thrombosis (2); infusion reaction (1); psychiatric (1). 
cohorts. $^{7-9}$ It should also be noted that based on the CARG risk score, our cohort was much frailer than the original CARG patient populations with more than half in the high-risk group. ${ }^{13,14}$ Moreover, we found that a senescence-associated, proinflammatory CK milieu, which was readily reversed by prephase treatment, was significantly associated with GA-defined, prognostically important geriatric impairments in function, mobility, and chemotherapy toxicity risk. While our sample size and event rate are too small to definitively associate GA meas-
A

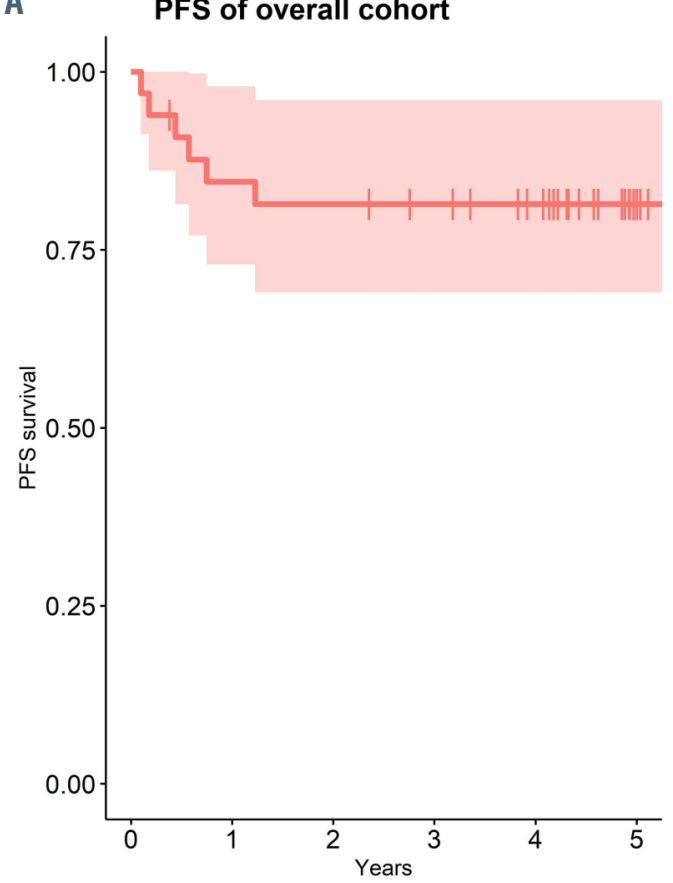

Number at risk

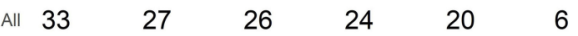

C

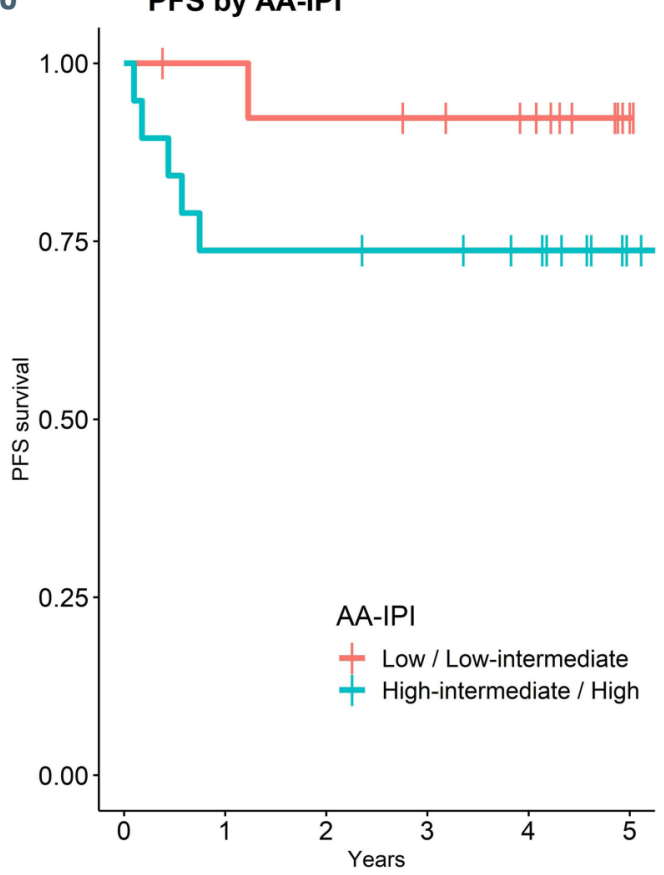

Number at risk

$\begin{array}{rrrrcr}-14 & 13 & 12 & 11 & 9 & 2 \\ -\quad 19 & 14 & 14 & 13 & 11 & 4\end{array}$

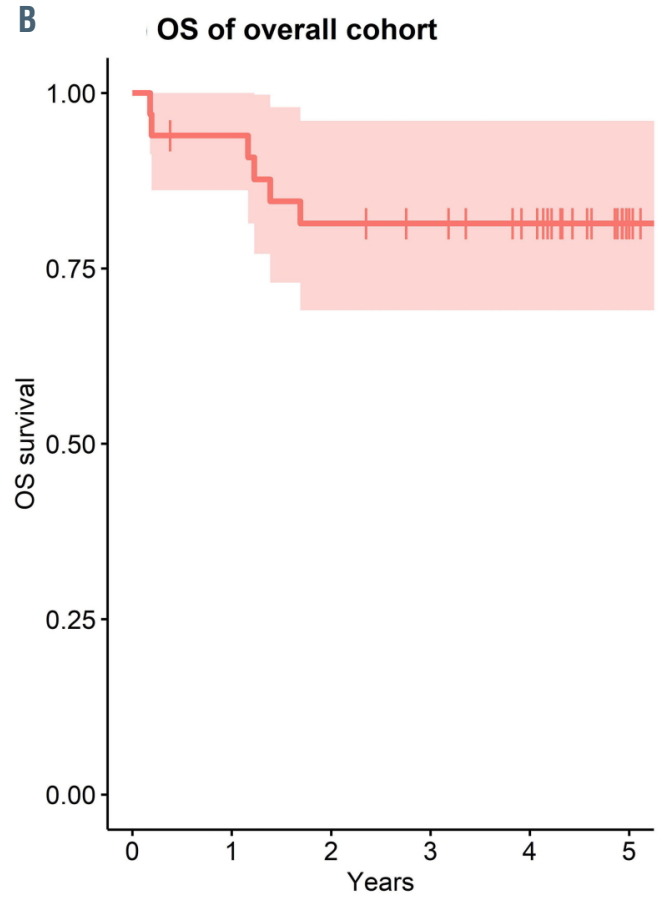

Number at risk
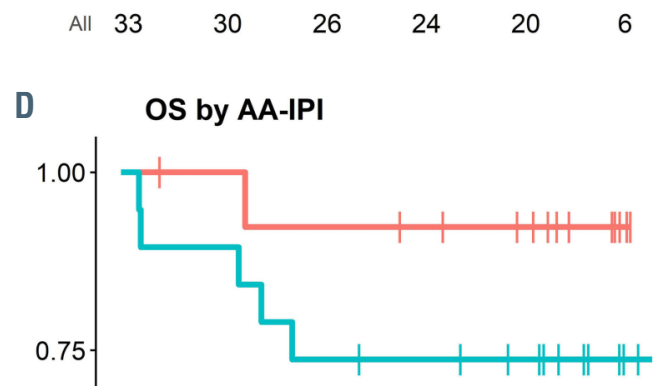

Figure 2. Survival outcomes. (A) Kaplan-Meier survival estimate of progression-free surviva (PFS) (red-line with shaded area denoted 95\% confidence interval). Life table is listed below. (B) KaplanMeier survival estimate of overall survival (OS) (redline with shaded area denoted $95 \%$ confidence interval). Life table is listed below. (C) Kaplan-Meier survival estimate of PFS by AA-IPI (red-line denoted low/low-intermediate and blue-line denoted highintermediate/high categories). Life table is listed below. (D) Kaplan-Meier survival estimate of OS by AA-IPI (red-line denoted low/low-intermediate and blue-line denoted highintermediate/high categories). Life table is listed below. 
ures and CK levels with survival, some of these measures may warrant additional investigation in larger cohorts of patients.

Since the NHL-02 trial, prephase therapy with vincristine and prednisone has since been adopted in several trials. ${ }^{7-9}$ It has been speculated that prephase therapy improves performance status thus reducing early cycle toxic death and allowing full-intensity chemoimmunotherapy as illustrated in the LYSA phase II trial. ${ }^{9} \mathrm{~A}$ recent prospective study also supported this notion by demonstrating an improved KPS and reduced incidence of febrile neutropenia with prephase therapy. ${ }^{22}$ However, the

Table 3. Impact of prephase therapy on geriatric assessment measures and proinflammatory cytokines.

\begin{tabular}{|c|c|c|c|c|}
\hline \multirow[b]{2}{*}{ Measures } & \multirow[b]{2}{*}{ N } & \multicolumn{3}{|c|}{ Pre- to post-prephase therapy changes } \\
\hline & & Changes (medlan, IQR) & P-value & q-value \\
\hline LDH (log level) & 30 & $0.01(-0.24,0.18)$ & 0.317 & 0.571 \\
\hline Clinician rated KPS & 32 & $0(0,10)$ & 0.039 & 0.198 \\
\hline Patient rated KPS & 32 & $0(0,10)$ & 0.420 & 0.621 \\
\hline $\mathrm{ADL}$ & 31 & $0(-10,7.5)$ & 0.483 & 0.621 \\
\hline IADL & 31 & $0(-0.5,0)$ & 0.637 & 0.717 \\
\hline Activity limitation & 31 & $0(-12.5,3.1)$ & 0.978 & 0.978 \\
\hline TUG & 29 & $-1(-2,1.01)$ & 0.105 & 0.304 \\
\hline CARG score & 32 & $0(-2,0)$ & 0.044 & 0.198 \\
\hline$\%$ risk & 32 & $0(-4,0)$ & 0.135 & 0.304 \\
\hline Cytokines (log level) & $\mathbf{N}$ & Changes (median, IQR) & & \\
\hline IFN- $\gamma$ & 30 & $-0.06(-1.61,0.85)$ & 0.208 & 0.416 \\
\hline IL-10 & 30 & $-0.5(-1.59,0.2)$ & 0.010 & 0.033 \\
\hline IL-12 & 30 & $-0.09(-0.38,0.27)$ & 0.306 & 0.439 \\
\hline IL-13 & 30 & $0(-1.19,1.95)$ & 0.514 & 0.643 \\
\hline IL1- $\beta$ & 30 & $0(0,1.19)$ & 0.953 & 0.953 \\
\hline IL-2 & 29 & $0.19(-0.39,1.68)$ & 0.890 & 0.953 \\
\hline IL-4 & 30 & $-0.25(-0.84,0.16)$ & 0.016 & 0.04 \\
\hline IL-6 & 30 & $-0.61(-1.49,0.05)$ & 0.002 & 0.01 \\
\hline IL-8 & 28 & $-0.33(-0.91,0.76)$ & 0.307 & 0.439 \\
\hline TNF- $\alpha$ & 30 & $-0.65(-1.49,-0.03)$ & 0.001 & 0.01 \\
\hline
\end{tabular}

IQR: interquartile range; LDH: lactate dehydrogenase; KPS: Karnofsky performance scale;ADL: activities of daily living; IADL: instrumental activities of daily living; TUG: timed-getup and go; CARG: cancer and aging research group; IFN: interferon; IL: interleukin; TNF: tumor necrosis factor.

Table 4. Association of baseline and changes in geriatric assessment measures with toxicity events.

\begin{tabular}{|c|c|c|c|c|c|}
\hline Characteristic (IQR) & N & At least 1 toxic event, $N=22$ & No toxic events, $N=11$ & P-value & q-value \\
\hline Baseline LOG LDH & 33 & $5.60(5.51,5.96)$ & $5.40(5.31,5.89)$ & 0.14 & 0.5 \\
\hline Baseline cKPS & 33 & $75(70,90)$ & $90(70,90)$ & 0.2 & 0.5 \\
\hline Baseline pKPS & 33 & $80(62,98)$ & $90(80,90)$ & 0.3 & 0.5 \\
\hline Baseline ADL & 33 & $48(26,84)$ & $80(32,92)$ & 0.13 & 0.5 \\
\hline Baseline IADL & 33 & $13.00(9.50,14.00)$ & $14.00(9.00,14.00)$ & 0.2 & 0.5 \\
\hline Baseline ACTIVITY LIMIT & 33 & $47(44,56)$ & $56(38,62)$ & 0.2 & 0.5 \\
\hline Baseline TUG & 31 & $11(10,20)$ & $11(9,13)$ & 0.2 & 0.5 \\
\hline Baseline CARG score & 33 & $10.50(9.00,13.00)$ & $9.00(7.00,10.50)$ & 0.040 & 0.4 \\
\hline Baseline \% risk & 33 & $66(54,89)$ & $54(52,54)$ & 0.019 & 0.3 \\
\hline$\Delta$ in LOG LDH & 30 & $0.01(-0.17,0.17)$ & $-0.20(-0.26,0.17)$ & 0.2 & 0.5 \\
\hline$\Delta$ in cKPS & 32 & $0(0,10)$ & $0(0,15)$ & 0.3 & 0.5 \\
\hline$\Delta$ in $p K P S$ & 32 & $0(0,10)$ & $0(0,5)$ & 0.6 & 0.7 \\
\hline$\Delta$ in ADL & 31 & $5(-5,10)$ & $0(-10,0)$ & 0.8 & 0.9 \\
\hline$\Delta$ in IADL & 31 & $0.00(-1.00,0.00)$ & $0.00(0.00,0.00)$ & 0.3 & 0.5 \\
\hline$\Delta$ in ACTIVITY LIMIT & 31 & $0(-14,6)$ & $0(-12,0)$ & 0.5 & 0.7 \\
\hline$\Delta$ in TUG & 29 & $-1.1(-4.2,1.1)$ & $-0.3(-1.4,0.8)$ & 0.7 & 0.8 \\
\hline$\Delta$ in CARG score & 32 & $0.00(-3.00,0.00)$ & $0.00(-1.00,0.50)$ & 0.8 & 0.9 \\
\hline$\Delta$ in $\%$ risk & 32 & $0(-12,0)$ & $0(0,0)$ & $>0.9$ & $>0.9$ \\
\hline
\end{tabular}

IQR: interquartile range; LDH: lactate dehydrogenase; cKPS: clinician-rated karnofsky performance scale; pKPS: patient-rated Karnofsky performance scale;ADL: activities of daily living; IADL: instrumental activities of daily living; TUG: timed-get-up and go; CARG: cancer and aging group. 


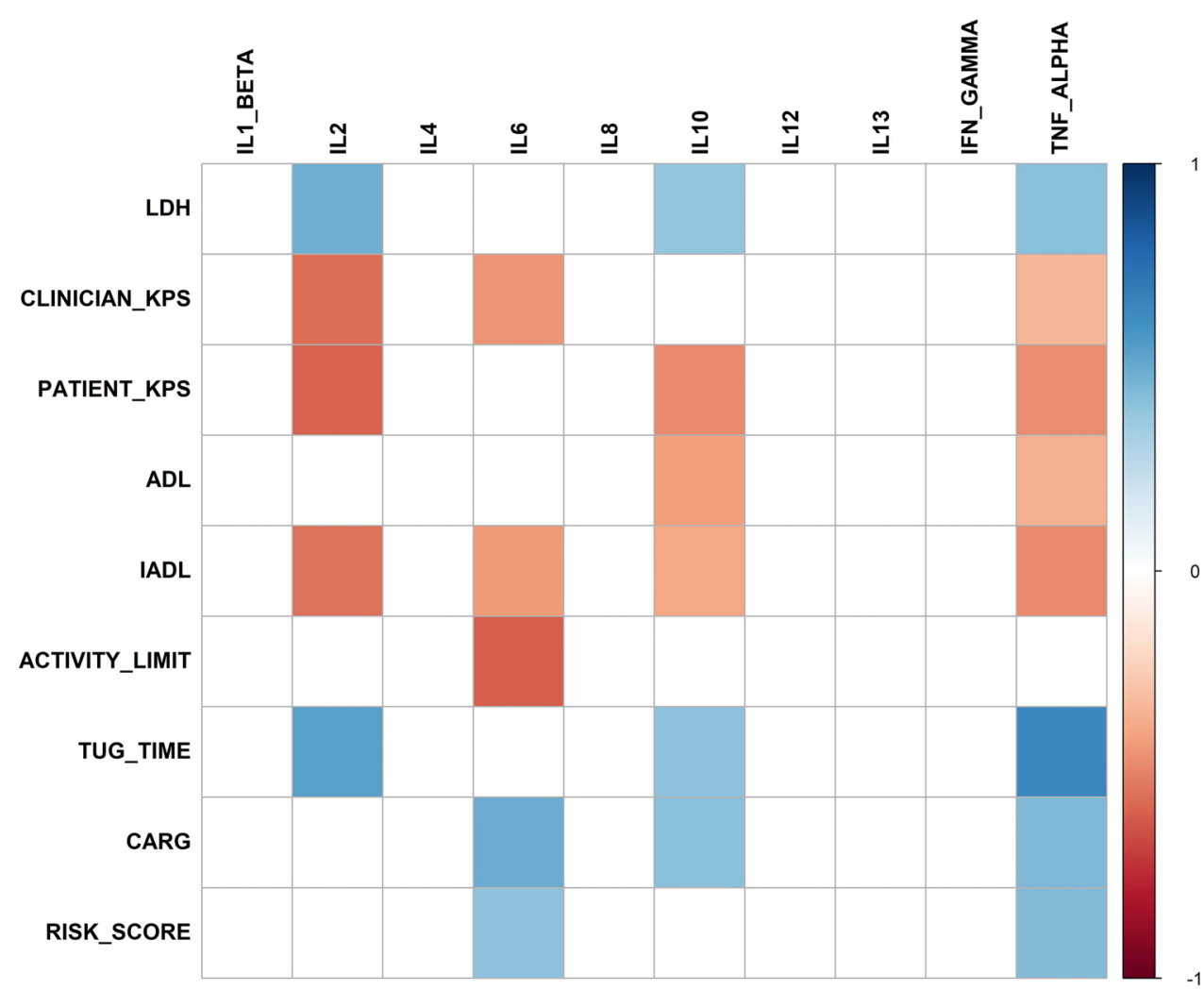

Figure 3. Heatmap of significant correlations of baseline geriatric assessment measures with baseline proinflammatory cytokine levels ( $q<0.05)$ with blue color demonstrating significant positive association and red color demonstrating significant negative association. LDH: lactate dehydrogenase; KPS: Karnofsky performance scale; ADL: activities of daily living; IADL: instrumental activities of daily living; TUG: timed-get-up and go; CARG: cancer and aging group; IFN: interferon; IL: interleukin; TNF: tumor necrosis factor.

underlying mechanism has never been examined and vincristine has significant neurotoxicity especially for older adult thus making it a poor partner of prednisone. ${ }^{10}$ Rituximab is likely more effective as a cytoreductive and debulking agent than vincristine at a dose of $1 \mathrm{mg}$; concurrently, however, we acknowledge concern for rituximab toxicities which include infusion related reactions that at times can be severe. We showed that this regimen led to an acceptable rate of $48 \%$ grade $3+$ non-hematologic plus grade $4+$ hematologic toxicities in this vulnerable cohort of older patients who were at high risk for chemotherapyrelated toxicities. Importantly, most patients in our cohort were able to complete planned cycles of curative, anthracycline-based chemoimmunotherapy and there was one early cycle death. Therefore, it is not surprising that our cohort of patients had outstanding PFS and OS. However, while we had hoped to see a decrease in mortality/toxicity from the prephase treatment, we could not directly compare survival and toxicity results from this pilot study with small sample size to previous large trials and registry studies. $^{7-9,22,23}$ In addition, with this design we cannot ascertain if rituximab's addition to prednisone added benefit, although the additional dose of rituximab did not appear to add toxicity.

Among hematologic malignancies including lymphoma, GA domains including function, mobility, cognition, and comorbidity have been consistently shown to be associated with survival and/or treatment-related toxicities. ${ }^{24-27}$ Our study is the first to examine specifically the CARG chemotherapy risk score in lymphoma patients and its longitudinal changes. The CARG score is easy to derive and well validated in solid tumors, however, its dynamic changes in response to therapy is unknown. ${ }^{13,14}$ In our study, we were unable to demonstrate a significant change in GA measures pre- and post-prephase therapy. It is possible that the short time interval, less than 2 weeks on average, is not adequate to detect a significant change in GA domains such as functional status. Indeed, a recent study in acute myeloid leukemia patients measured GA changes 8-12 weeks following the initial assessment. ${ }^{28}$ Alternatively, our sample size may be too small or that prephase treatment alone is inadequate to impact GA changes. Interestingly, there was a suggestion of an association between the baseline CARG risk score and the absolute chemotherapy toxicity risk with TE, which will be explored further. Nevertheless, given accumulating evidence of how geriatric frailty affects older lymphoma patients, ${ }^{29,30}$ our longitudinal GA data may allow in-depth examination of the prognostic impact of both baseline and changes in individual geriatric deficits, and may also be used prospectively to guide treatment-decision making in older, vulnerable lymphoma patients as shown in previous studies. ${ }^{31-34}$

Perhaps the most interesting aspect of our findings is the SASP-associated, proinflammatory cytokine milieu and its relationship to GA and prephase therapy. A few key cytokines in this age-related SASP such as IL-6, TNF$\alpha$

IL-10, and IL-2 were strongly associated with geriatric impairments including functional limitations in 
ADL/IADL, mobility impairment measured by TUG, and CARG chemotherapy toxicity risk score. This finding suggests a novel, chronic inflammation-based, biologic underpinning of geriatric frailty in older lymphoma patients and several potential mechanism-based intervention strategies. Most importantly, this proinflammatory cytokine milieu was readily reversed with prephase therapy which may account for its effectiveness. Some of these cytokines were also associated with baseline lactate dehydrogenase, suggesting that tumor burden may also contribute to geriatric frailty and the SASP, consistent with a previous study showing that tumor debulking could improve lymphoma patients' KPS. ${ }^{35}$ We could not, however, ascertain the contribution of disease-related factors in our study. Nevertheless, there are several additional implications. First, if validated, these specific cytokines such as TNF- $\alpha$, IL- 6 , and IL-10 could potentially serve as frailty biomarkers for older lymphoma patients. This notion is supported by previous findings where IL- 6 and TNF- $\alpha$ were found to be associated with KPS, cytopenia, $\mathrm{OS}$, and PFS. ${ }^{36-38}$ These markers are biologically and mechanistically more specific than c-reactive protein or albumin. ${ }^{9,39}$ It is also possible that elevated proinflammatory cytokines are caused by a combination of disease, comorbidities, KPS, and/or other host factors, in addition to cellular senescence. These possibilities will need to be examined in a large study. Second, the use of senolytics to deplete senescence cells is a potential strategy that could potentially reverse the SASP-associated frailty phenotype in the short term. Commercially available senolytics such as desatinib/quercetin have been studied in an early phase human trial. ${ }^{40,41}$ Finally, specific inhibitors of these frailty $\mathrm{CK}$ are available commercially. One of them, siltuximab, has been tested in a phase I trial for patients with hematologic malignancies including NHL. The drug was well tolerated with no dose-limiting toxicity and sustained suppression of CRP was observed. ${ }^{42}$ Although we examined most key inflammatory cytokines, not all SASP-associated proteins were analyzed in this study and thus our results may not be generalizable to other components of SASP.

In conclusion, we show that rituximab/prednisone is a feasible and safe prephase regimen, which may have enhanced the delivery of chemoimmunotherapy for older, vulnerable patients with newly diagnosed DLBCL. Our findings of acceptable short-term toxicities and excellent long-term survival are hypothesis generating and mechanistically appealing since this strategy appears to target the aging-related SASP and the proinflammatory CK milieu. While requiring validation from a prospective randomized study which should also examine individual components of the prephase regimen, we propose that prephase therapy with rituximab/prednisone is considered for older, vulnerable NHL patients starting curative-intent chemoimmunotherapy. We also strongly advocate for incorporating GA into the care of older lym- phoma patients given its wealth of prognostic information and potential value in improving treatment tolerance.

\section{Disclosures}

RJL sits on the advisory board for Kite, a Gilead Company; $P A H$ has received research support and consultancy fees from Portola Pharmaceuticals, Inc.; AN has received research funding from Pharmacyclics and Raphael, honoraria from Prime Oncology, Medscape and EUSA, and sits on the advisory Board for Janssen; ADZ has received consultancy fees from Genentech/Roche, Gilead, Celgene, Janssen, Amgen, Novartis and Adaptive Biotechnology, has received research funding from MEI Pharmaceuticals, Roche, Gilead and Beigene, sits on the advisory Board for MorphoSys, Gilead, Genentech, Abbvie and AstraZeneca, serves as DMC chair for Beigene and is DMC member for BMS/Celgene/Juno; MJM has receieved consultancy fees or sits on the advisory boards of Genentech, Roche, GlaxoSmithKline, Bayer, Merck, Pharmacyclics, Janssen, Seattle Genetics, Takeda, Teva, Juno Therapeutics, Rocket Medical, June Therapeutics, Immunovaccine Technologies, and Daiichi Sankyo, has received research funding from Genentech Roche, GlaxoSmithKline, IGM Biosciences, Bayer, Pharmacyclics, Janssen, Rocket Medical, Seattle Genetics, and Immunovaccine Technology; GAS acts as a consultant and sits on the advisory board for Abbvie, Allogene, Autolus, Beigene, BMS/Celgene, Debiopharm, Genmab, Kite/Gilead, Janssen, Milteniy, Morphosys, Norvartis, Roche, and Velosbio. All other authors declare no conflict of interests.

\section{Contributions}

$P A H, C N O, R J L$, and $B K$ designed the research, interpreted the data, and wrote the paper; RJL,CNO, AI, MO,DLS, and $E D$ collected and analyzed the data; $P A H, C N O, A D Z, A N$, DJS, SMH, AJM, PCC, AMH, AK, MJM, MLP, CLB, AY, $J F G$, and PRD contributed the data. All authors reviewed and approved the manuscript.

\section{Acknowledgements}

We acknowledge administrative assistance provided by Shreena Patel.

\section{Funding}

This research was supported in part by the NIH/NCI Cancer Center Support grant P3O CA008748 and the Lacher Lymphoma Foundation. The content is solely the responsibility of the authors and does not necessarily represent the official views of the National Institutes of Health.

\section{Data sharing statement}

Deidentified individual participant data that underlie the reported results will be made available 3 months after publication for a period of 5 years after the publication date upon request. The study protocol is included as a data supplement available with the online version of this article.

\section{References}

1. Hamlin PA, Satram-Hoang S, Reyes C, Hoang KQ, Guduru SR, Skettino S. Treatment patterns and comparative effectiveness in elderly diffuse large B-cell lymphoma patients: a surveillance, epidemiology, and end results-medicare analysis.
Oncologist. 2014;19(12):1249-1257

2. Levin E, Peng Y, Ji Y, Gilbertson D, Morrison VA. Practice patterns in older patients with diffuse large B-cell lymphoma: a medicare analysis, 2007-2015. J Geriatr Oncol. 2020;11(8):1344-1348.

3. Mareschal S, Lanic H, Ruminy P, Bastard C, Tilly $\mathrm{H}$, Jardin $\mathrm{F}$. The proportion of activated B-cell like subtype among de novo dif- fuse large B-cell lymphoma increases with age. Haematologica. 2011;96(12):18881890.

4. Soubeyran PL, Cordoba R. Approaches for vulnerable and frail older patients with diffuse large B-cell lymphomas. Curr Opin Oncol. 2019;31(5):369-373.

5. Khan Y, Brem EA. Considerations for the treatment of diffuse large B cell lymphoma 
in the elderly. Curr Hematol Malig Rep. 2019;14(4):228-238.

6. Morrison VA, Hamlin P, Soubeyran P, et al. Approach to therapy of diffuse large B-cell lymphoma in the elderly: the International Society of Geriatric Oncology (SIOG) expert position commentary. Ann Oncol. 2015;26(6):1058-1068

7. Pfreundschuh M, Trumper L, Kloess M, et al. Two-weekly or 3-weekly CHOP chemotherapy with or without etoposide for the treatment of young patients with good-prognosis (normal LDH) aggressive lymphomas: results of the NHL-B1 trial of the DSHNHL. Blood. 2004;104(3):626-633.

8. Pfreundschuh M, Schubert J, Ziepert M, et al. Six versus eight cycles of bi-weekly CHOP-14 with or without rituximab in elderly patients with aggressive CD20+ Bcell lymphomas: a randomised controlled trial (RICOVER-60). Lancet Oncol. 2008; 9(2):105-116.

9. Peyrade F, Bologna S, Delwail V, et al. Combination of ofatumumab and reduceddose CHOP for diffuse large B-cell lymphomas in patients aged 80 years or older: an open-label, multicentre, single-arm, phase 2 trial from the LYSA group. Lancet Haematol. 2017;4(1):e46-e55.

10. Madsen ML, Due H, Ejskjaer N, Jensen P, Madsen J, Dybkaer K. Aspects of vincristine-induced neuropathy in hematologic malignancies: a systematic review. Cancer Chemother Pharmacol. 2019; 84(3):471-485.

11. Mohile SG, Dale W, Somerfield MR, et al. Practical assessment and management of vulnerabilities in older patients receiving chemotherapy: ASCO Guideline for Geriatric Oncology. J Clin Oncol. 2018; 36(22):2326-2347.

12. DuMontier C, Loh KP, Bain PA, et al. Defining undertreatment and overtreatment in older adults with cancer: a scoping literature review. J Clin Oncol. 2020; 38(22):2558-2569.

13. Hurria A, Togawa K, Mohile SG, et al. Predicting chemotherapy toxicity in older adults with cancer: a prospective multicenter study. J Clin Oncol. 2011;29(25):34573465.

14. Hurria A, Mohile S, Gajra A, et al. Validation of a prediction tool for chemotherapy toxicity in older adults with cancer. I Clin Oncol. 2016;34(20):23662371.

15. Lin RJ, Behera M, Diefenbach CS, Flowers $\mathrm{CR}$. Role of anthracycline and comprehensive geriatric assessment for elderly patients with diffuse large B-cell lymphoma. Blood. 2017;130(20):2180-2185

16. Lopez-Otin C, Blasco MA, Partridge L, Serrano M, Kroemer G. The hallmarks of aging. Cell. 2013;153(6):1194-1217.

17. Gorgoulis V, Adams PD, Alimonti A, et al. Cellular senescence: defining a path forward. Cell. 2019;179(4):813-827.
18. Tchkonia T, Zhu Y, van Deursen J, Campisi J, Kirkland JL. Cellular senescence and the senescent secretory phenotype: therapeutic opportunities. J Clin Invest. 2013; 123(3):966-972

19. Faget DV, Ren Q, Stewart SA. Unmasking senescence: context-dependent effects of SASP in cancer. Nat Rev Cancer. 2019; 19(8):439-453

20. Katzman R, Brown T, Fuld P, Peck A, Schechter R, Schimmel H. Validation of a short Orientation-Memory-Concentration Test of cognitive impairment. Am Psychiatry. 1983;140(6):734-739.

21. Guigoz Y, Vellas B. The Mini Nutritional Assessment (MNA) for grading the nutritional state of elderly patients: presentation of the MNA, history and validation. Nestle Nutr Workshop Ser Clin Perform Programme. 1999;1:3-11; discussion 11-12.

22. Lakshmaiah KC, Asati V, Babu KG, et al Role of prephase treatment prior to definitive chemotherapy in patients with diffuse large B-cell lymphoma. Eur J Haematol. 2018;100(6):644-648

23. Juul $M B$, Jensen $P H$, Engberg $H$, et al. Treatment strategies and outcomes in diffuse large B-cell lymphoma among 1011 patients aged 75 years or older: a Danish population-based cohort study. Eur J Cancer. 2018;99:86-96.

24. Klepin HD. Ready for prime time: role for geriatric assessment to improve quality of care in hematology practice. Blood. 2019; 134(23):2005-2012

25. Abel GA, Klepin HD. Frailty and the management of hematologic malignancies. Blood. 2018;131(5):515-524.

26. Liu MA, DuMontier C, Murillo A, et al. Gait speed, grip strength, and clinical outcomes in older patients with hematologic malignancies. Blood. 2019;134(4):374-382.

27. Hshieh TT, Jung WF, Grande LJ, et al. Prevalence of cognitive impairment and association with survival among older patients with hematologic cancers. JAMA Oncol. 2018;4(5):686-693.

28. Saad M, Loh KP, Tooze JA, et al. Geriatric assessment and survival among older adults receiving postremission therapy for acute myeloid leukemia. Blood. 2020; 136(23):2715-2719

29. Tucci A, Martelli M, Rigacci L, et al. Comprehensive geriatric assessment is an essential tool to support treatment decisions in elderly patients with diffuse large B-cell lymphoma: a prospective multicenter evaluation in 173 patients by the Lymphoma Italian Foundation (FIL). Leuk Lymphoma. 2015;56(4):921-926.

30. Merli F Luminari S, Rossi G, et al Outcome of frail elderly patients with diffuse large Bcell lymphoma prospectively identified by Comprehensive Geriatric Assessment: results from a study of the Fondazione Italiana Linfomi. Leuk Lymphoma. 2014; 55(1):38-43.
31. Bai JF, Han HX, Feng R, et al. Comprehensive geriatric assessment (CGA): a simple tool for guiding the treatment of older adults with diffuse large B-cell lymphoma in China. Oncologist. 2020;25(8):e1202-e1208.

32. Olivieri A, Gini G, Bocci C, et al. Tailored therapy in an unselected population of 91 elderly patients with DLBCL prospectively evaluated using a simplified CGA. Oncologist. 2012;17(5):663-672.

33. Spina M, Balzarotti M, Uziel L, et al. Modulated chemotherapy according to modified comprehensive geriatric assessment in 100 consecutive elderly patients with diffuse large B-cell lymphoma. Oncologist. 2012;17(6):838-846

34. Merli F, Luminari S, Tucci A, et al Simplified geriatric assessment in older patients with diffuse large B-cell lymphoma: the Prospective Elderly Project of the Fondazione Italiana Linfomi. J Clin Oncol. 2021;39(11):1214-1222.

35. Bowcock SJ, Fontana V, Patrick HE. Very poor performance status elderly patients with aggressive B cell lymphomas can benefit from intensive chemotherapy. $\mathrm{Br}$ Haematol. 2012;157(3):391-393.

36. Voog E, Bienvenu J, Warzocha $\mathrm{K}$, et al Factors that predict chemotherapy-induced myelosuppression in lymphoma patients: role of the tumor necrosis factor ligandreceptor system. J Clin Oncol. 2000, 18(2):325-331.

37. Dlouhy I, Filella X, Rovira J, et al. High serum levels of soluble interleukin-2 receptor (sIL2-R), interleukin-6 (IL-6) and tumor necrosis factor alpha (TNF) are associated with adverse clinical features and predict poor outcome in diffuse large B-cell lymphoma. Leuk Res. 2017;59:20-25

38. Zhong H, Chen J, Cheng S, et al. Prognostic nomogram incorporating inflammatory cytokines for overall survival in patients with aggressive non-Hodgkin's lymphoma. EBioMedicine. 2019:41:167-174

39. Loh KP, Tooze JA, Nicklas BJ, et al Inflammatory biomarkers, geriatric assessment, and treatment outcomes in acute myeloid leukemia. J Geriatr Oncol. 2020; 11(3):410-416.

40. Justice JN, Nambiar AM, Tchkonia T, et al Senolytics in idiopathic pulmonary fibrosis Results from a first-in-human, open-label, pilot study. EBioMedicine. 2019;40:554563.

41. Chen Y, Liu S, Leng SX. Chronic low-grade inflammatory phenotype (CLIP) and senescent immune dysregulation. Clin Ther. 2019;41(3):400-409.

42. Kurzrock R, Voorhees PM, Casper C, et al A phase I, open-label study of siltuximab, an anti-IL-6 monoclonal antibody, in patients with B-cell non-Hodgkin lymphoma, multiple myeloma, or Castleman disease. Clin Cancer Res. 2013;19(13):36593670 